\title{
Interactive comment on "Coupling urban drainage-wastewater systems and electric smart grids during dry periods: a gain/loss framework using the relative economic value with ensemble flow forecasts to predict the switch" by Vianney Courdent et al.
}

Anonymous Referee \#1

Received and published: 4 November 2016

Review comments on manuscript hess-2016-522 Coupling urban drainage-wastewater systems and electric smart grids during dry periods: A gain/loss framework using the relative economic value with ensemble flow forecasts to predict the switch between management objectives

General comments

The manuscript presents an interesting topic within the scope of the journal. While 
enough information on the used methodology is given to enable a rough understanding, the reader will not be able to reproduce the applied methods based on that information. Result interpretation will be difficult for the reader without more specific information on the used hydrological model (its calibration and accuracy) and the WWTP and the potential impact of the described method on its performance in terms of energy consumption and effluent water quality. More information in the results section on the performance of the proposed method and a discussion of its risks and benefits would be very desirable as without them it is difficult to draw clear conclusions about the value of the method. For these reasons, I suggest to accept the paper for a major revision thereby also giving the authors the opportunity to address a number of structural deficiencies and language related details.

\section{Specific comments}

P1 L1: This is one long title. Would "Coupling urban drainage-wastewater systems and electric smart grids during dry periods" not suffice? Even then I find the title somewhat misleading: Is the WWTP not continuously coupled to the smart grid? If I understand correctly, rather than coupling and uncoupling, only the temporal amount of energy consumption is optimized depending on the hydraulic condition.

P2 L8ff: Maybe I misunderstand this sentence, but it seems to suggest that only because rain only occurs $7 \%$ of the time it makes sense to look into energy optimization. Would it make less sense if you had $10 \%$ of rain? Could you clarify/rephrase this?

P2 L8ff: Dry weather flow rarely can be defined by "no rain", usually (sufficient sewer network size and event intensity) wet weather conditions will be predominant for several hours after a rain event has ended (as the proposed method does by using the flow rather than the rain as a trigger for switching between control objectives). I suggest rephrasing this section accordingly.

P2 L10ff (same phrase again, sorry): Striving for energy optimization and emission reduction of WWTP is standard practice for many years - both during dry and wet

Printer-friendly version

Discussion paper

$\mathrm{C2}$ 
weather. With this background in mind it would be better to slightly rephrase and cite some relevant literature here to avoid this phrase being interpreted as a novel suggestion as such.

P3 L15ff: Some details are discussed here that re-occur in section 3. Delete here?

P4 L5: A clear(er) definition of REV (as eg in the abstract) would be critical here as it forms the basis of the paper

P5 L20ff: Are methods (i) and (ii) defined in literature? They seem to be a mix of other methods. It would not be possible to reproduce your method from this section. Please give more specific information on the used methods for enhancing the forecast.

P6 section 2 and especially 2.2 discusses methods. I'd suggest to move these parts to section 3

P6 L20ff: You mention that the DWF module of the model is calibrated, but there are no details on the calibration of the Nash cascades. These details seem essential for accurate predictions, please add them to this section.

P5, section 2.3: No details on the WWTP and its energy consumption are given while this forms the focus of the paper. Changed flow regimes at the influent will necessarily cause changes in energy consumption of the WWTP. How are these considered in this study?

P6 L31ff: Move to introduction?

P7 L5ff: Leave out? Does not add anything to the understanding of the reader.

P11 L5ff: are these not results that should be moved to the next section?

Figure 8 is not interpreted in the text. Omit?

Printer-friendly version

P12 L13: How big are the uncertainties in the energy price forecasts? Could you comment on their (potential) influence on your method? 
P12 L15ff: In this scenario, it is possible to save $26 € M W h$ during 2 days. I suggest to add information that answers at least a number of the following questions in order for the reader to be able to understand the meaning of this result: Is this a representative result? What is the average/peak energy consumption of the WWTP? What is the maximum amount of energy that could be switched? How much could be saved during the 2 years of data you used? How does that impact the total energy consumption of the WWTP ('switching' could result in an increase as well as a decrease)? What is the influence on the WWTP effluent performance and emission of greenhouse gasses?

P12 L15ff: What is the cost of the suggested system (at the least the NWP data will have to be purchased + some man-hours for keeping the real-time system up and running) as compared to its benefits? It seems these considerations should be included in order to judge the actual gains produced by the system.

P12 L24 "optimization based on economic objectives will also yield environmental benefits": This seems a much too broad statement that should be explained or based on a citation. In the context of this paper, it seems that it would be perfectly possible to create a scenario where 'switching' energy consumption would lead to an overall increase of energy consumption (e.g. by running the blowers on a frequency at which they are less efficient than when not 'switching'), but a decrease in cost.

P12 L26: "most EMs predict the high flow event but often too early" Is this a problem of the precipitation prediction or the hydrological model? This would be difficult for the reader to judge (without information on the calibration of the hydrological model). Maybe you could add a line in figure 9: the output of the hydrological model given the observed rain. This would also (more or less) address the above comment for P6 L20ff.

Technical corrections

Printer-friendly version

P2 L18: “... consumption thought bids" $\rightarrow$ “... consumption through bids"

P2 L27 “... is also influence by..." -> “... is also influenced by..."

Discussion paper 
P3 L1 “... detain ...” $\rightarrow$ “...store..."

P3 L22 "The development of ... models have led..." $\rightarrow$ "The development of ... models has led..."

P3 L26 "...decreasing special grid size..." -> "...decreasing spatial grid size..."

P3 L27 "...under dispersive..." -> "...underdispersive..."

P4 L8: “...the used of post-processing ...” $\rightarrow$ “...the use of post-processing ...”

P4 L10: “...energy marked ..." -> “...energy market ...”

P6 L4: “...circles of Figure 3 which...” -> “...circles of Figure 3), which...”

P8 L18:"...member..." -> “...members..."

P9 L8: “...on ...” $\rightarrow$ omit

P9 L16: "...loss associated these events..." -> "...loss associated with these events..."

P9 L19 “...event...” -> “...events...”

P10 L21: "...the forecast in beneficial..." -> "...the forecast is beneficial..."

P11 L7: “...high a negative impact..." -> "...a high negative impact..."

P12 L2: “...the need an economical..." -> “...the need for an economical..."

Figure 6: In 6 a 4 corresponds to red and 5/25. $6 \mathrm{~b}: 4$ is red and 16/25. Is this correct? What is "legend 5" in Figure 6a?

Figure 9, uppermost left ordinate: should this not be "energy price"?

P14 L30 "This is underling..." -> "This underlines..."?

Interactive comment on Hydrol. Earth Syst. Sci. Discuss., doi:10.5194/hess-2016-522, 2016. 